\title{
Are Incisional and Excisional Skin Tension Lines Biomechanically Different? Understanding the Interplay between Elastin and Collagen during Surgical Procedures
}

\author{
Sharad P. Paul* \\ School of Medicine, University of Queensland; Faculty of Surgery, University of \\ Auckland; Auckland University of Technology; New Zealand
}

\begin{abstract}
Background: Since Langer's first foray into studying cutaneous lines, although people have studied skin lines across the body, there has not been a study that elucidates changes to skin structure of elastin and collagen at different load levels. This study set out to look at whether incisional lines and excisional lines have different biodynamics and have to be considered differently.

Materials and Methods: For this study, we used a two-photon microscopic camera using optimal wavelengths to detect collagen and elastin. Measurements were taken in 5 patients at the center of the excisional wound (high-load) and at the ends of the wound (tapered end of the ellipse) where effectively the wound is an incisional wound.

Results: Wounds were observed after they were surgically closed. When incisional wounds were observed, where there was minimal tension $(<1.5 \mathrm{~N}$ force $)$ we found that, in each case, elastin stretched and collagen buckled, revealing mostly elastin. Where larger defects were created after excisions (as in the figure where a skin cancer had been removed, where forces to close wounds were typically greater than $2 \mathrm{~N}$ ), we noted that the image revealed mostly collagen, suggesting that the reverse had occurred (i.e. collagen stretched and elastin buckled).

Conclusion: This difference between tension loads on skin and the interplay between collagen and elastin has never before been elucidated for incisional and excisional wounds, and in the author's view has great research interest for a cutaneous surgeon seeking to identify the best skin lines to utilize to minimize scarring. (International Journal of Biomedicine. 2017;7(2):111-114.)
\end{abstract}

Key Words: skin lines $\bullet$ collagen $\bullet$ elastin $\bullet$ skin tension $\bullet$ surgery $\bullet$ excisions $\bullet$ incisions $\bullet$ keloid $\bullet$ scarring

\section{Introduction}

Elastin, as the name indicates, makes skin elastic providing the organ with the ability to stretch and recoil. ${ }^{(1)}$ Collagen in skin responds to mechanical forces by altering its molecular structure and generates biochemical signals to influence wound healing and tissue remodeling. ${ }^{(2)}$ Levels of collagen and elastin change with the depth of the dermis and age - and in the lower dermis a significant difference between young and old has been noted for elastin with varying collagen/ elastin ratios. ${ }^{(3)}$ Since Langer's seminal work in 1861, his 'cleavage lines of skin' ended up de facto surgical lines, even

*Corresponding author: Dr. Sharad P. Paul. Senior Lecturer, School of Medicine, University of Queensland; Senior Lecturer, Faculty of Surgery, University of Auckland; Adjunct Professor, Auckland University of Technology.E-mail: sharad@sharadpaul.com for most surgical excisions of skin lesions. ${ }^{(4,5)}$ It has already been suggested by others that in the trunk and limbs, Langer's lines predominantly align with elastin fibers. ${ }^{(6)}$

After Langer marked out cleavage lines by using a round-tipped cutting instrument and noting the direction the clefts elongated, Kocher, in 1892, suggested that these lines be used for surgical procedures. ${ }^{(7)}$ It has been noted in recent times that incisions placed at right angles to the direction of skin cleavage lines had a higher risk of hematoma and tension, and thereby a higher risk of hypertrophic scarring. (8) But the mechanisms of wound tension, especially when defects are created due to removal of skin lesions, have been poorly understood. Biomechanical studies have shown that skin behaves elastically only at low-load levels. For example, on the feet, due to weight-bearing tissues, where the load increases skin reveals increased viscoelastic behavior (i.e. strain becomes a function of load and time) ${ }^{(9)}$ While it is well 
known that skin is anisotropic, it also exhibits orthotropy (i.e. a degree of symmetry with regard to two normal planes, especially in regions of the body close to bone). ${ }^{(10)}$ One of the theories has been that this is due to a preferential orientation of collagen fibers..$^{(11)}$

Incisions made along the long-axis of Langer's skin cleavage lines are essentially lines in the direction of minimum skin extensibility, and in areas like the calf $76 \%$ of elastin fibers aligned themselves along Langer's lines. ${ }^{(12)}$ Yet, we also know from other studies that in the superficial dermis, collagen fibers are not oriented along cleavage lines and it is the reticular dermis that determines skin anisotropy. ${ }^{(13)}$ However, the precise roles of elastin and collagen during skin stretch or load for excisional surgery are unknown. In this article, the author studies differences between incisional and excisional skin wounds to try and understand whether the roles of elastin and collagen under low- and high-tension loads differ.

\section{Materials and Methods}

For this study, we used a two-photon microscopic camera using optimal wavelengths to detect collagen and elastin. Previous studies have detected collagen and elastin in skin using fluorescence imaging using specific emission wavelengths: for collagen (lambda c) at $380 \mathrm{~nm}$, and for elastin $450 \mathrm{~nm}$, using excitation wavelengths (lambda e) of $340 \mathrm{~nm}$, and $380 \mathrm{~nm}$, respectively. ${ }^{(14)}$ Therefore, these parameters were used as a guide in planning for this study. The study was undertaken in 5 patients (age range 25-74) who were undergoing cutaneous surgery for skin cancer. Measurements were taken at the center of the excisional wound (high-load) and at the ends of the wound (tapered end of the ellipse) where effectively the wound is an incisional wound. Using a two-photon microscopic camera (developed in-house in conjunction with Shenzhen Do3think Technology Co., Ltd), measurements were taken of incisional and excisional sites in each case (Fig.1).
Under a spectroscope, elastin is similar to collagen with an absorption peak around $320 \mathrm{~nm}$ and an emission peak near $400 \mathrm{~nm}$. Collagen can similarly be viewed, using a SHG microscopic camera, where two photons combine from the laser field to produce a scattered photon of exactly half the wavelength. ${ }^{(15)}$ The study showed that second-harmonic generation signals derived from collagen can be spectrally isolated from elastin two-photon fluorescence. Two-photon fluorescence signals can be further characterized by emission maxima at $495 \mathrm{~nm}$ and $520 \mathrm{~nm}$, corresponding to elastin and cellular contributions, respectively. This method may not be strictly fluorescence, but is very useful to visualize collagen and elastin separately. Others have also noted that secondharmonic generation microscopy has emerged as a powerful modality for imaging fibrillar collagen in a diverse range of tissues, including skin. ${ }^{(16)}$

Alongside confocal microscopy, two-photon microscopy is now also being used to detect skin cancer, and some authors have successfully used this method to monitor collagen remodeling in vivo after micro-ablative fractional laser resurfacing. ${ }^{(17)}$

\section{Results}

Wounds were observed after they were surgically closed, as shown in the image. When incisional wounds were observed, where there was minimal tension $(<1.5 \mathrm{~N}$ force $)$ we found that, in each case, elastin stretched and collagen buckled, revealing mostly elastin. Where larger defects were created after excisions (as in the figure where a skin cancer had been removed, where forces to close wounds were typically greater than $2 \mathrm{~N}$ ), we noted that the image revealed mostly collagen, suggesting that the reverse had occurred (i.e. collagen stretched and elastin buckled).

Multi-photon microscopy has found favor as a technique to elucidate elastin and collagen in tissues. ${ }^{(18)}$
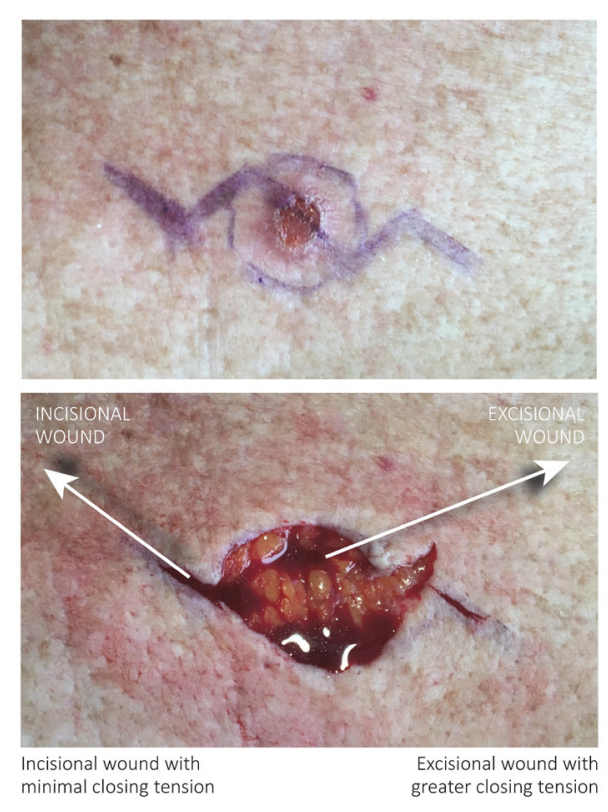

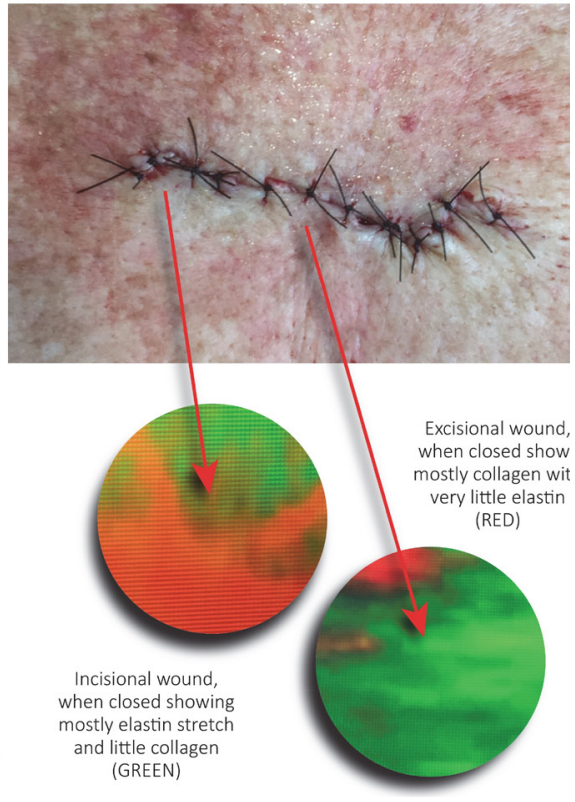

Fig. 1. Interplay between collagen and elastin in surgical wounds. 
The main advantage of multi-photon microscopy is based on two-photon excited fluorescence (TPEF) and secondharmonic generation (SHG) imaging, allowing us to observe non-fixed, unstained tissue samples. Because collagen in the skin dermis can easily produce an SHG signal and elastin is more effective in generating TPEF, multi-photon microscopy has found widespread applications in dermatology for studying and differentiating cutaneous collagen and elastin - in general, using this technique the collagen images are color-coded in green and elastin images are color-coded in red to increase the contrast. ${ }^{(18,19)}$

The findings were similar and in fact virtually identical in all cases. The images demonstrating a typical finding (Fig.1) show that for incisional lines (low tension wounds) elastin stretches and collagen buckles, and for excisional wounds (after removal of skin lesions) it is predominantly collagen at play with very little elastin involvement. This not only confirms our contention regarding biodynamic excisional skin tension (BEST) lines ${ }^{(20)}$ that we must view skin lines differently for incisions and excisions, but we see this as the starting point for more research into wound, scar and skin dynamics after excisional surgery. This is why this study set out to look at the roles of elastin and collagen in surgical wounds when closing incisions (inherently low-load) or excisions (higher-loads due to larger defects).

Our finding that incisional (low-load) wound closures are primarily under the influence of elastin, and excisional wound (higher-load) closures depend on collagen has implications for research into both wound healing and scar formation.

\section{Discussion}

This difference between tension loads on skin and the interplay between collagen and elastin has never before been elucidated between incisional and excisional wounds, and in the author's view has great research interest for a cutaneous surgeon seeking to identify the best skin lines to utilize to minimize scarring. However, until now there has been no attempt to differentiate incisional and excisional skin lines.

We contend that therefore we need to view incisional and excisional wounds differently and has been mapping best excisional skin tension (BEST) lines. ${ }^{(20)}$ Human skin, when viewed as a mere physical membrane ends up with skin lines and wrinkles because a keratinocyte-stiffened epidermis drapes a softer and thicker dermis. Of course, anatomical sites like knees and elbows have wrinkles that can be considered 'tension' wrinkles (two-dimensional, due to geometry, pretension and joint action) and in other areas like the forehead, muscle action causes 'compression' wrinkles (one-dimensional due to muscle action only), but in our view, BEST lines for surgical excisions may not be along these lines. ${ }^{(21)}$ Add to this, other's findings that in keloid scars the increase of both elastin and collagen occurs in deep dermis, whereas a sharp decrease of elastin is found in the upper dermis of keloid, ${ }^{(22)}$ and we have the beginnings of new insights and research into cutaneous surgery and wound healing.

Keloids are unique to humans with no comparable animal models. ${ }^{(3)}$ Researchers have found significant differences in the morphology and content of collagen and elastin in the upper dermis and deep dermis of keloid tissue. In the upper dermis, elastin is not very visible and in the lower dermis, elastin is abundant. Given the findings in this study that incisional wounds are full of elastin and excisional wounds are filled with collagen, further avenues for research into skin lines used during surgery and the resultant scars beckon. We also know from studies into aging that changes as a function of the depth of dermis are significant only for elastin for both young and old. ${ }^{(3)}$ The lower dermis is less rich in elastin, and shows significant diminution between young and older age groups. ${ }^{(3)}$ This also has implications for suture placement in incisional and excisional wounds. This study also demonstrates the use of multi-photon microscopy for assessing the morphology and quantity variations of collagen and elastin in incisional and excisional wounds, a technique used by others to study keloid scarring, ${ }^{(18)}$ and this study is a starting point for further research to understand the basic science behind surgical wounds we create, and resultant scar formation - as ultimately, it is the latter that patients worry about the most.

\section{Conflicts of interest}

There are no commercial interests or conflicts of interest to declare.

\section{Acknowledgement}

This paper resulted from my $\mathrm{PhD}$ research project at the University of Queensland's School of Medicine and I would like to acknowledge my supervisors, Assoc. Professor Cliff Rosendahl, University of Queensland, and Professor John Windsor, University of Auckland. I would also like to thank Ryan Butler, Auckland University of Technology, for his help with illustrations.

\section{References}

1. Almine JF, Bax DV, Mithieux SM, Nivison-Smith L, Rnjak J, Waterhouse A, et al. Elastin-based materials. Chem Soc Rev. 2010;39(9):3371-9. doi: 10.1039/b919452p.

2. Chang S-W, Buehler M.J. Molecular biomechanics of collagen molecules. Mater Today. 2014;17(2).

3. Pittet J-C, Freis O, Vazquez-Duchêne M-D, Périé G, Pauly G. Evaluation of Elastin/Collagen Content in Human Dermis in-Vivo by Multiphoton Tomography --Variation with Depth and Correlation with Aging. Cosmetics. 2014;1(3):21121. doi:10.3390/cosmetics 1030211

4. Langer K. On the anatomy and physiology of the skin I. The cleavability of the cutis. Br J Plast Surg.1978;31(1):3-8

5. Waldorf JC, Perdikis G, Terkonda SP. Planning incisions. Oper Tech Gen Surg.2002;4(3):199-206.

6. Zahouani H, Djaghloul M, Vargiolu R, Mezghani S, Mansori MEL. Contribution of human skin topography to the characterization of dynamic skin tension during senescence: morpho-mechanical approach. Journal of Physics: Conference Series. 2014;483:012012. doi:10.1088/17426596/483/1/012012

7. Kocher E.T. Chirurgische Operationslehre. Jena, Fischer; 1892. 
8. Motegi K. Consideration of the formation and biological significance of hypertrophic scar. J Maxillofac Surg. 1984;12(3):123-7.

9. Reihsner R, Balogh B, Menzel E J. Two-dimensional elastic properties of human skin in terms of an incremental model at the in vivo configuration. Med Eng Phys. 1995;17(4):304-13.

10. Lanir Y, Fung YC. Two-dimensional mechanical properties of rabbit skin. I. Experimental system. J Biomech. 1974; 7(1):29-34.

11. Lanir Y, Fung YC. Two-dimensional mechanical properties of rabbit skin. II. Experimental results. $J$ Biomech.1974;7(2):171-82.

12. Manschot J, Wijn P, Brakkee A. The angular distribution function of elastic fibres as estimated from in vivo measurements. In: Huiskes R, van Campen DH, de Wijn JR, editors. Biomechanics. Vol. I: Principles and Applications. M. Nijhoff, The Hague; 1982

13. Agache PG, Humbert P. Measuring the skin: Non-invasive investigations, physiology, normal constants. Berlin: Springer; 2004.

14. Tang J, Zeng F, Savage H, Ho PP, Alfano RR. Fluorescence spectroscopic imaging to detect changes in collagen and elastin following laser tissue welding. J Clin Laser Med Surg. 2000;18(1):3-8.

15. Mertz J, Moreaux L. Second-harmonic generation by focused excitation of inhomogeneously distributed scatterers. Opt. Commun. 2001;196:325-30.

16. Chen X, Nadiarynkh O, Plotnikov S, Campagnola PJ. Second harmonic generation microscopy for quantitative analysis of collagen fibrillar structure. Nat Protoc. 2012;7(4):654-69. doi: 10.1038/nprot.2012.009.

17. Cicchi R, Kapsokalyvas D, Troiano M, Campolmi P, Morini C, Massi D, et al. In vivo non-invasive monitoring of collagen remodelling by two-photon microscopy after micro-ablative fractional laser resurfacing. J Biophotonics. 2014;7(11-12): 914-25. doi: 10.1002/jbio.201300124.

18. Chen J, Zhuo S, Jiang X, Zhu X, Zheng L, Xie S, Lin B, Zeng H. Multiphoton microscopy study of the morphological and quantity changes of collagen and elastic fiber components in keloid disease. J. Biomed. Opt. 2011;16(5):051305. doi: $10.1117 / 1.3569617$.

19. Riemann I., Alexander Ehlers A, LeHarzic R, Martin S, Reif A, König K. In vivo multiphoton tomography of skin during wound healing and scar formation. Proc. SPIE 6442, Multiphoton Microscopy in the Biomedical Sciences VII; 644226 (February 10, 2007); doi:10.1117/12.702407

20. Paul SP, Matulich J, Charlton N. A New Skin Tensiometer Device: Computational Analyses To Understand Biodynamic Excisional Skin Tension Lines. Sci. Rep. 2016;6:30117. doi: 10.1038/srep30117.

21. Paul SP. The Epistemology of Wrinkles: From Geology and Anatomy to Physiology. International Journal of Biomedicine. 2016;6(3):237-9.

22. Chen J, Zhuo S, Jiang X, et al. Multiphoton microscopy study of the morphological and quantity changes of collagen and elastic fiber components in keloid disease. J Biomed. Opt. 2011;16(5): 051305. doi: 10.1117/1.3569617. 